\title{
Overcoming classical measurement limits through entangle- ment in photon number: an introduction
}

\author{
M.Genovese ${ }^{1, \mathrm{a}}$ and G.Adenier ${ }^{1}$, D.Calonico ${ }^{1}$, I.P.Degiovanni ${ }^{1}$, S.Micalizio ${ }^{1}$, I.Ruo Berchera ${ }^{1}$, \\ P.Traina ${ }^{1}$ \\ ${ }^{1}$ INRIM, Strada delle Cacce 91, I-10135 Torino, Italy
}

\begin{abstract}
We discuss the recent developments in exploiting Quantum Optical Correlations in order to overcome the classical limits on measurement. After a general introduction to the argument, we will consider in detail some specific and emblematic protocols, and focus in particular on applications to the holometer.
\end{abstract}

\section{Introduction}

The powerful properties of quantum states [1] have opened the possibility of performing tasks beyond the limits set by classical theories, thereby blooming in a vast field collectively christened as quantum technologies. Among these technologies, quantum metrology and quantum imaging are of particular interest as they can improve the sensitivity and the resolution of measurements, by exploiting nonclassical features such as entanglement and squeezing. Here, we present some recent developments in overcoming classical limits by exploiting Quantum Optical Correlations (QOC) in photon number [2-11]. We will briefly review two protocols exploiting photon number correlation in order to detect either the absorption or the reflection of objects with enhanced sensitivity. Then, we will consider in detail an application to a double interferometric scheme aiming at detecting the ultra-faint phase signal that could emerge from quantum geometry at Planck's scale.

\section{Quantum imaging protocols based on photon number correlations}

A natural application in which strong photon number correlation can be helpful is the measurement of the absorption (reflection) coefficient of objects, when the shot noise level - i.e. the unavoidable level of intensity fluctuation for classical states of light (equal to $\sqrt{N}$ where $N$ is the number of photons utilized in the measurement) — is the bottleneck that limits the accuracy of the measurement.

\subsection{Sub-Shot-Noise Imaging}

The first implementation that we briefly mention $[2,3]$ is the image reconstruction of a weak absorbing object with a signal-to-noise ratio below the shot noise level. This imaging technique is based on the measurement of the photon number difference $N_{1}-N_{2}$ between pairs of correlated spatial modes,

ae-mail: m.genovese@inrim.it 
obtained by parametric down conversion (PDC) and detected by means of the pixels of a CCD camera. The idea is that the noise-free image of the object can be recovered by subtracting from the noisy image, obtained in one branch of the PDC, the correlated noise pattern in the other branch. The performance is directly related to the noise reduction factor $\sigma \equiv\left\langle\delta^{2}\left(N_{1}-N_{2}\right)\right\rangle /\left\langle N_{1}+N_{2}\right\rangle \approx 1-\eta$ $(\delta N=N-\langle N\rangle)$ depending on the detection probability $\eta[2,9,12-17]$. The method is effective for values of detection efficiency that are easily achievable, as demonstrated by our experiment.

In principle, Fock states $|n\rangle$ can be used as well for sub-shot-noise imaging by simply measuring the number of detected photons $\left\langle N_{\alpha(\mathbf{x})}\right\rangle$ after the object, where $\alpha(\mathbf{x})$ is the absorption in the transverse spatial coordinate $\mathbf{x}$. It is easy to show that the sensitivity of the measurement defined in the usual way as $\alpha_{\min }=\left\langle\delta^{2} N_{\alpha}\right\rangle /\left\langle\partial_{\alpha} N_{\alpha}\right\rangle$ in the limit of $\alpha \rightarrow 0$ is given by the Fano factor of the detected light, i.e. $\alpha_{\text {min }} \simeq\left\langle\delta^{2} N\right\rangle /\langle N\rangle$. In particular starting from a Fock state $|n\rangle$ and considering the detection efficiency $\eta$, we have

$$
\alpha_{\min } \simeq(1-\eta),
$$

as in the case of QOC, while classically the Fano factor is lower bounded by the unity. However, the actual realization of Fock states is extremely challenging ${ }^{1}$, and therefore the photon number correlations, as in QOC, represent the only viable solution to adopt in practice.

Spontaneous PDC would be an obvious choice for generating photon number QOC if it was not, in general, too faint in intensity to be useful in most of the possible applications, for instance in biology. Even though the uncertainty is improved by a factor $(1-\eta)$ with respect to the shot noise limit $1 / \sqrt{N}$, in most of the cases the same improvement can be obtained by just increasing the number of photons $N$, while still remaining far from the limits imposed, for example, by the damaging threshold of the sample. It would therefore be relevant to make QOC available at the same intensity as used in real applications.

For this purpose, a source of correlated beams exploiting four wave mixing (4WM) in hot atomic vapor is a good candidate [19-22]. Four-wave mixing is indeed a known source of intense nonclassical twin beams: in fact, the first experimental demonstration of squeezed light was actually made using $4 \mathrm{WM}$ with sodium atoms [23]. 4WM is a nonlinear phenomenon in optical media with third order susceptibility $\chi^{(3)}$. It can be generated when an intense laser beam (the pump) and a weak laser beam (the seed) overlap in a $\chi^{(3)}$ medium (in our case cesium vapor [24]), with frequencies close to resonance with the relevant atomic transitions. The interest for this type of source to generate twin beams has been renewed recently. The effective Hamiltonian describing the 4WM process is similar to that of SPDC, but it does not suffer from some drawbacks of SPDC in terms of conversion efficiency and collection efficiency of the pair of generated photons. Indeed, 4WM is not hindered by the inherently low efficiency that characterizes the conversion of pump photons into daughter photons in SPDC. The twin beams generated by $4 \mathrm{WM}$ are in fact not only bright but also narrowly defined in terms of frequency and spatial modes, instead of being spread out in direction and frequency as with SPDC. There is thus no need to restrict further the collection of detected photons with spatial and frequency filtering, a necessity that makes the collecting of SPDC photon pairs an even more inefficient process than it already is. Another advantage is that the twin beams generated by $4 \mathrm{WM}$ have frequencies naturally close to atomic transitions, so that $4 \mathrm{WM}$ is well suited for atom-light interaction and atom-based quantum protocols.

The configuration that we aim for here is when the frequencies of the pump and the seed reach a double- $\Lambda$ configuration with three atomic levels (see Figure 1), so that when two pump photons are absorbed, the seed (signal) is amplified and a conjugate (idler) is generated. The effective Hamiltonian of the process takes the form $\hat{\mathcal{H}}=\gamma\left[\hat{a}_{\mathrm{s}} \hat{a}_{\mathrm{i}}+\hat{a}_{\mathrm{s}}^{\dagger} \hat{a}_{\mathrm{i}}^{\dagger}\right]$, where the labels of the creation and annihilation operators stand for signal and idler. The $4 \mathrm{WM}$ process is efficient when $\omega_{\text {conj }}=\omega_{\text {seed }}+2 \delta$.

\footnotetext{
${ }^{1}$ Recent developments in emission from next color centers in diamond are good candidates to proceed in this direction [18].
} 


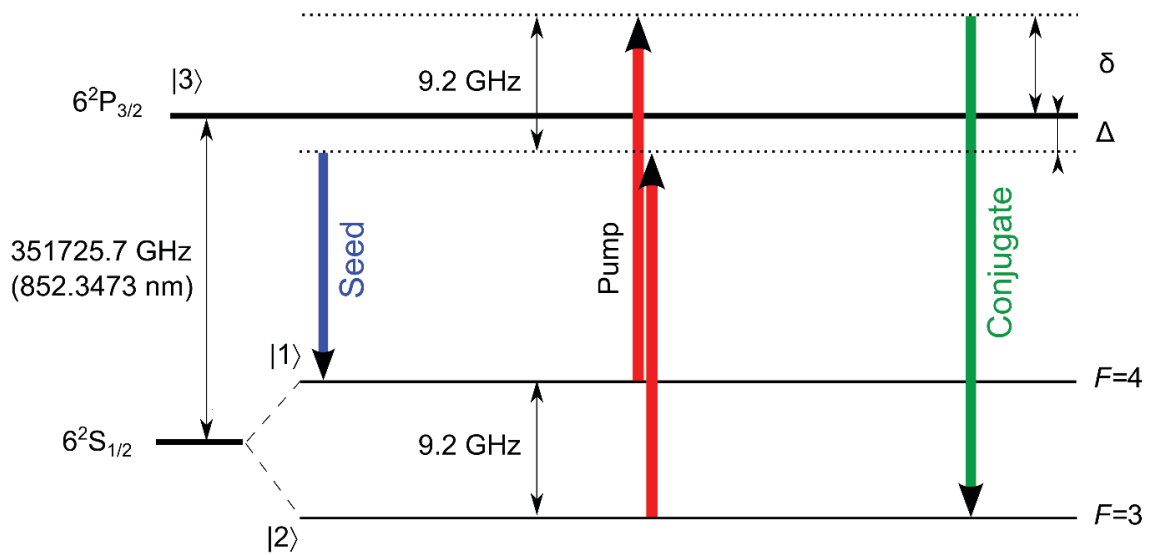

Figure 1. Double- $\Lambda$ configuration for $4 \mathrm{WM}$ with the $D_{2}$ line of ${ }^{133} \mathrm{Cs}$, over a three-level system consisting of two lower levels and one upper level. The lower levels are the hyperfine splitting of $6^{2} S_{1 / 2}$, with $F=3$ and $F=4$, and are labelled respectively $|1\rangle$ and $|2\rangle$. The upper level is $6^{2} P_{3 / 2}$, labelled $|3\rangle$. The $4 \mathrm{WM}$ resonance is obtained when the frequency difference between the pump and the seed matches the frequency difference between the two lower level, that is, when it is close to $\delta=9.2 \mathrm{GHz}$.

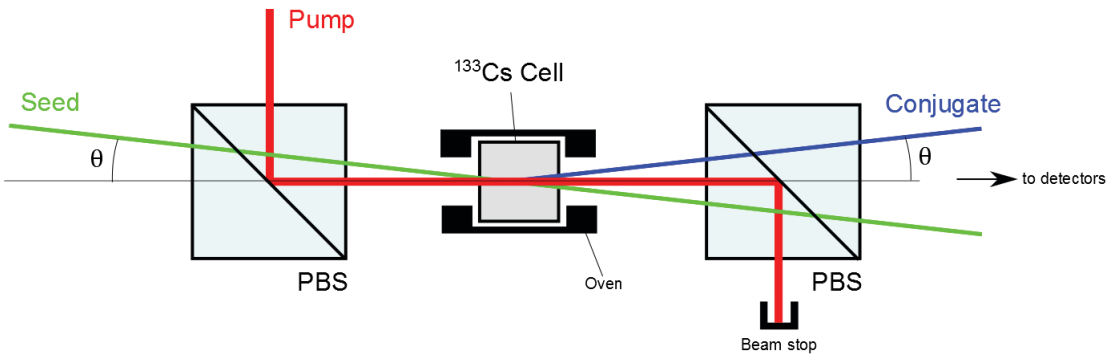

Figure 2. Experimental setup for $4 \mathrm{WM}$ in the non-colinear case. The pump (red) and the seed (green) are orthogonally polarized and overlap in a cell of cesium vapor placed in an oven, thereby generating a conjugate beam (blue), together with an amplification of the seed (green). The pump is removed by its orthogonal polarization with the use of a PBS.

Using the following notations: $\mu$ being the number of photons per mode of the seed, $\lambda$ the number of photons per mode of the spontaneous emission, and $\eta$ the detection efficiency (assumed to be equal in both channels), one can write the parametric amplifier equations as: $\left\langle N_{1}\right\rangle=\eta \lambda(1+\mu)$ for the conjugate beam, and $\left\langle N_{2}\right\rangle=\eta \mu+\eta \lambda(1+\mu)$ for the seed beam. The quantum noise reduction between the two beams is

$$
\frac{V\left(N_{1}-N_{2}\right)}{\left(N_{1}+N_{2}\right)}=1-\eta+\frac{\eta}{2 G-1},
$$

when $\mu \gg 1$ and $\mu \gg \lambda$, where $G=1+\lambda$ is the gain of the $4 \mathrm{WM}$ process. 
In order to implement such a $4 \mathrm{WM}$ process, we use a cell containing ${ }^{133} \mathrm{Cs}$ vapor, of $1 \mathrm{~cm}$ long, maintained at 110 degrees Celcius. Both the intense pump beam and the seed beam are produced by CW semi-conductor diode lasers with $852 \mathrm{~nm}$ wavelength. They are overlapping inside the cell, with orthogonal polarization in order to eliminate the pump at the exit of the cell (see Figure 2). The frequencies of the lasers are tuned and stabilized by laser controllers (temperature and current controllers). In our setup, the pump is currently limited to $110 \mathrm{~mW}$, with $200 \mathrm{~mm}$ waist, whereas the seed is maintained at about $10 \mu \mathrm{W}$ with $50 \mathrm{~mm}$ waist.

The preliminary results obtained with our source show that the 4WM signal can be observed over a wide range of 1-photon detuning from the $D_{2}$ lines. The highest gain that we observed (see Figure 3 ) is obtained for a negative detuning (i.e., detuned to the red) of $-2.2 \mathrm{GHz}$ with respect to the transition with higher frequency. With such rather large detuning, the pump is relatively less absorbed and depolarized, so that the the spatial and polarization modes of the pump are better preserved and easier to discriminate from the seed and conjugate, both in the colinear and non-colinear configuration. The frequency of the seed and of the conjugate are also benefiting from such a large 1-photon detuning, as they are amplified and emitted with frequencies away from the $D_{2}$ absorption lines, which should preserve a good intensity correlation between them.

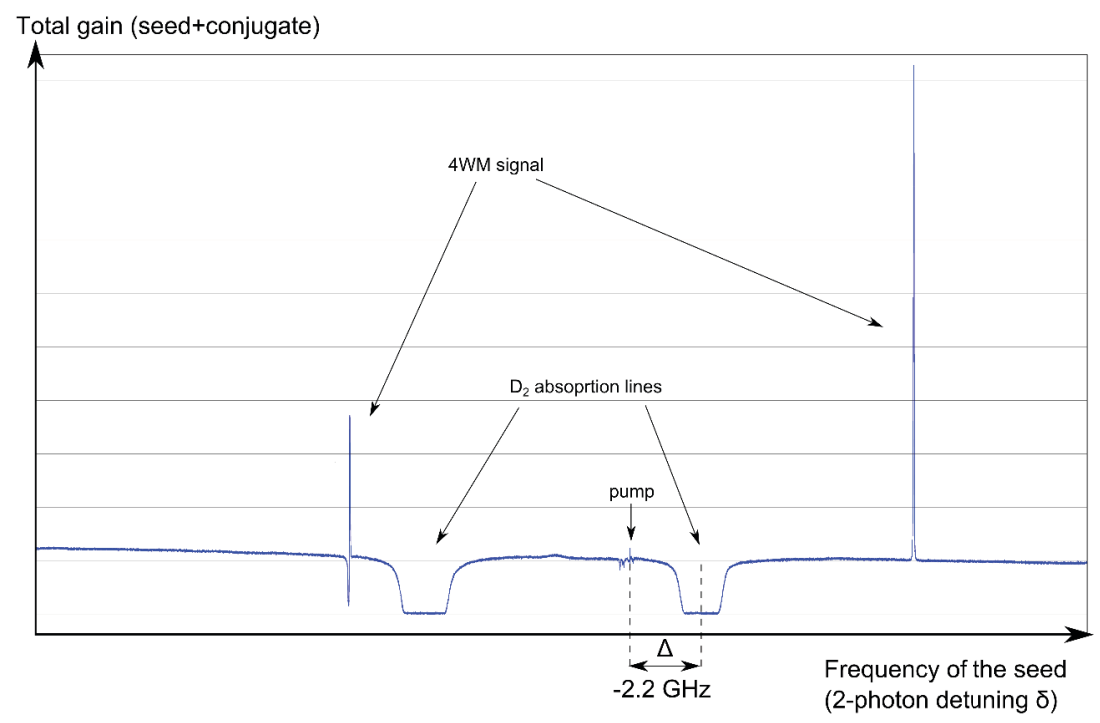

Figure 3. Sweeping of the frequency of the seed around to the $D_{2}$ lines of ${ }^{133} \mathrm{Cs}$, for a fixed frequency of the pump, in the colinear configuration. The highest gain is obtained when the pump is detuned (single-photon detuning $\Delta$ ) to the red of the $D_{2}$ line with higher frequency.

An important effect that we observed is that the transitions to hyperfine levels are shifted by the intense pump, presumably due to light-shift effect (a.c. Stark shift). Consequently, the optimum frequency difference between the seed and the pump is not always exactly equal to the $9.193 \mathrm{GHz}$ of the hyperfine splitting of $6^{2} S_{1 / 2}$ that one would expect to lead to an efficient $4 \mathrm{WM}$ process, but it depends on the relative shift induced by the pump on these hyperfine levels.

While the optimum gain is obtained in the colinear case, as the region of interaction of the overlapping beam is then maximized, the configuration that ultimately interests us for QOC is the noncolinear case, as it gives spatially distinct beams for the seed and the conjugate, whose strong cor- 


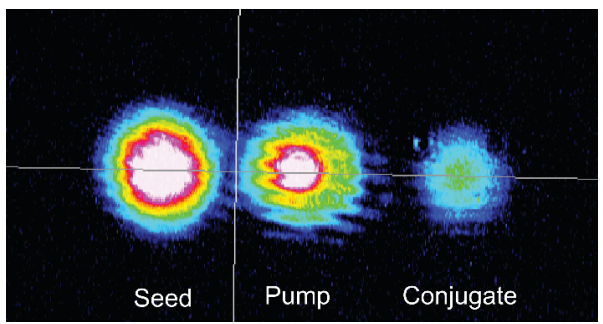

Figure 4. Non-colinear 4WM with the pump allowed to partially pass through (for demonstration purpose). The pump can be discriminated and eliminated by its polarization, as it is orthogonal to that of the seed and the conjugate.

relation can then be utilized. In the non-colinear configuration, the seed is tilted with a small angle from the pump inside the cell, which allows spatially discriminating the three output beams from one another. With the large (and negative) single-photon detuning used here, the pump retains well its spatial coherence and polarization, and can thus be easily discriminated from the seed and the conjugate, which are bright and narrowband beams that are promising for QOC (see Figure 4). The next step will consist in observing sub-shot noise with those beams by sending them to separate and balanced detectors, and by subtracting the photocurrents to measure a noise of less magnitude than that of a coherent state of the same power.

\subsection{Quantum enhanced detection of reflective target in a noisy environment}

Although the aim of optical quantum metrology is to improve the sensitivity and/or the resolution of measurements exploiting nonclassical state of light and correlations, in most of the realistic scenarios, the intrinsic difficulties in realizing such states, in particular the typical level of losses and noise, are known to nullify the advantage of adopting quantum strategies.

In [4] we presented a striking application of quantum correlation for metrology, designed to target detection in a noisy environment, where the limitations mentioned above were partially overcome. The principle of these techniques is to take advantage of the photon number second order correlation and/or of the entanglement of two conjugated branches of parametric down conversion. Remarkably, our scheme preserves a strong advantage over the best classical counterparts even in the presence of large amounts of noise and losses. Here we provide a quick presentation of the idea and the main results. For more details see [5], and for further developments and applications see [25].

The protocol is inspired by the twin beams based "Quantum Illumination" elaborated in [6], although our analysis considers in particular reception strategy based on photon-counting detection and measurement of the covariance $\left\langle\delta N_{1} \delta N_{2}\right\rangle$. One beam (probe) is sent towards a partially reflecting object and collected back, while the correlated beam (ancilla) does not interact with the target and is used for the joint measurement. A strong background (typically a thermal beam) impinges the detector together with the probe. Its photons are regarded as indiscernible from the photons of the probe.

The quantum enhancement in terms of signal-to-noise ratio (SNR) with respect to the classical case $(\mathrm{CL})$ can be directly expressed by the value of the generalised Cauchy-Schwartz parameter $\varepsilon \equiv$ 
$\left\langle: \delta N_{1} \delta N_{2}:\right\rangle /\left(\left\langle: \delta^{2} N_{1}:\right\rangle\left\langle: \delta^{2} N_{2}:\right\rangle\right)^{1 / 2}$ evaluated for the twin beam state

$$
\varepsilon=S N R_{Q O C} / S N R_{C L}=\mu /(1+\mu),
$$

which only depends on the mean number of photon per spatio-temporal mode $\mu$. Losses and noise background do not affect the quantum enhancement.

The improvement with respect to the classical case is also related to the mutual information of the twin beams which exceeds the one of classically correlated beams [7]. This application does not require entanglement, however it relies on non-classical correlations in the sense of the non-classicality of the Glauber-Sudarshan $P$ function as demonstrated from the fact that the Cauchy-Schwartz parameter is always $\varepsilon>1$.

This protocol could very possibly pave the way for adopting quantum correlation in realistic scenarios, and challenges the common belief that their use is always limited by their fragility with respect to noise and losses.

\section{Quantum light for testing Quantum geometry: the holometer}

The dream of building a theory unifying general relativity and quantum mechanics, the so-called quantum gravity (QG), has been a key element in theoretical physics research for the last 60 years. This achievement would represents indeed the ultimate goal of the research addressed to build a "theory of everything", describing all the components and interactions of the physical world (the last of "Einstein's dream"). It would therefore be of the utmost interest not only for the whole physics community, but also for philosophers and scientists of other disciplines. Furthermore, its impact on the world vision of mankind can only be presumed to be of the utmost importance.

Several attempts in this direction have been considered. However, for many years, no testable prediction emerged from these studies, leading to the common wisdom that this kind of research belonged more to the field of mathematics rather than physics, being by construction unable to produce experimentally testable predictions as required by Galilean scientific method.

In the last few years this common wisdom has been challenged [26-32]. A first series of testable proposals concerned photons propagating over cosmological distances [26-28], with the problem of extracting QG effects from a limited (and uncontrollable) observational sample affected by various propagation effects. More recently, effects in interferometers connected to non-commutativity of position variables in different directions [29-31] have been considered both for cavities with microresonators [29] and for an apparatus based on two coupled interferometers [30, 31], as in the case of the so-called "holometer", i.e. a double 40 m interferometer at Fermilab.

The idea at the heart of the holometer is that non-commutativity at the Planck scale $\left(1.616 \times 10^{-35} \mathrm{~m}\right)$ for position variables in different directions $[33,34]$ leads to an additional phase noise, called holographic noise (HN). In a single interferometer this noise substantially confounds with other sources of noise, even for the most sensitive gravitational wave interferometers [30, 31], since their HN resolution is worse than their resolution to gravitational-wave at low frequencies. Nonetheless, if the two equal interferometers of the holometer have overlapping spacetime volumes, then the HN between them is correlated and easier to be identified [30, 31]. In order to check that the observed correlation is really due to the holographic noise, one needs to compare a configuration where the arms of the two interferometers are parallel (overlapping spacetime volumes) with a configuration where two arms are perpendicular (non-overlapping spacetime volumes), since in this last case no holographic noise should appear. The ultimate limit for holometer sensitivity, as for any classical-light based apparatus, is dictated by the shot noise: therefore, the possibility of going beyond this limit by exploiting quantum optical states is of the greatest interest $[2,35]$ and, due to the considerations of the previous section, one could expect that QOC can help in this sense. 
In the past, the possibility of exceeding shot-noise limit in gravitational-wave detectors was suggested [36, 37] and recently implemented [38] by using squeezed light (see also the recent LIGO result [39]). This prompts the possibility of greatly improving quantum gravity tests by exploiting quantum correlated light.

Indeed, in [8] we performed a theoretical study addressed to verify the advantages of quantum light in a double interferometer [40]. The results of our work clearly demonstrate that both the use of squeezed light and of twin beams present a large advantage for sufficiently good transmission and detection efficiencies. In particular the advantage of twin beam, i.e. exploiting entanglement as a resource, can become really large for high efficiencies.

In general, the observable measured at the output of the holometer may be described by an appropriate operator $\widehat{C}\left(\phi_{1}, \phi_{2}\right), \phi_{k}$ being the phase shift (PS) detected by the interferometer $\mathcal{I}_{k}, k=1,2$. Its expectation value is $\left\langle\widehat{C}\left(\phi_{1}, \phi_{2}\right)\right\rangle=\operatorname{Tr}\left[\rho_{12} \widehat{C}\left(\phi_{1}, \phi_{2}\right)\right]$, where $\rho_{12}$ is the overall density matrix associated with the state of the light beams injected in $\mathcal{I}_{1}$ and $\mathcal{I}_{2}$.

In order to observe the HN, one should compare $\left\langle\widehat{C}\left(\phi_{1}, \phi_{2}\right)\right\rangle$ in two different experimental configurations of $\mathcal{I}_{1}$ and $\mathcal{I}_{2}$, namely, parallel, "\|l", and perpendicular, " $\perp$ ". In the "\|l" configuration, the interferometers are oriented so that the HN induces the same random fluctuation of their PSs, leading to a substantial correlation between them, since they occupy overlapping space-time volumes. Thus, by measuring the correlation of the interference fringes, one can highlight the presence of the HN. The " $\perp$ " configuration serves as a reference measurement. It corresponds to the situation in which the correlation due to $\mathrm{HN}$ is absent, since the space-time volumes are not overlapping. In other words, it is equivalent to the estimation of the "background".

The statistical properties of the PSs fluctuations due to HN may be described by a suitable probability density function, $f_{x}\left(\phi_{1}, \phi_{2}\right), x=\|, \perp$. In turn, the expectation of any operator $\widehat{O}\left(\phi_{1}, \phi_{2}\right)$, or function of the PSs, should be averaged over $f_{x}$, namely,

$$
\left\langle\widehat{O}\left(\phi_{1}, \phi_{2}\right)\right\rangle \rightarrow \mathcal{E}_{x}\left[\widehat{O}\left(\phi_{1}, \phi_{2}\right)\right] \equiv \int\left\langle\widehat{O}\left(\phi_{1}, \phi_{2}\right)\right\rangle f_{x}\left(\phi_{1}, \phi_{2}\right) \mathrm{d} \phi_{1} \mathrm{~d} \phi_{2} .
$$

Since in the holometer the HN arises as a correlation between two phases, the covariance can be estimated by measuring the difference between the expectation value of the operator $\widehat{C}$ in parallel and perpendicular configurations.

One has to reduce as much as possible the uncertainty associated with these measurement:

$$
\mathcal{U}\left(\delta \phi_{1} \delta \phi_{2}\right) \approx \sqrt{\frac{\operatorname{Var}_{\|}\left[\widehat{C}\left(\phi_{1}, \phi_{2}\right)\right]+\operatorname{Var}_{\perp}\left[\widehat{C}\left(\phi_{1}, \phi_{2}\right)\right]}{\left[\left\langle\partial_{\phi_{1}, \phi_{2}}^{2} \widehat{C}\left(\phi_{1,0}, \phi_{2,0}\right)\right\rangle\right]^{2}}}, \quad\left(\delta \phi_{1}, \delta \phi_{2} \ll 1\right)
$$

where $\operatorname{Var}_{x}\left[\widehat{C}\left(\phi_{1}, \phi_{2}\right)\right] \equiv \mathcal{E}_{x}\left[\widehat{C}^{2}\left(\phi_{1}, \phi_{2}\right)\right]-\mathcal{E}_{x}\left[\widehat{C}\left(\phi_{1}, \phi_{2}\right)\right]^{2}$.

Since the holometer couples two interferometers, minimizing the noise on their outputs correlation, that is, regardless of the noise in the single interferometer, this suggests that QOC may enhance the performance of the holometer. Therefore, we consider a configuration like the one depicted in Fig. $5 \mathrm{a}$ where the two modes are excited in a continuous-variable maximally entangled state, i.e. a twomode squeezed vacuum state, or twin-beam state, $|\mathrm{TWB}\rangle\rangle_{a_{1}, a_{2}}=S_{12}(\zeta)|0\rangle_{a_{1}, a_{2}}=\sum_{n=0}^{\infty} c_{n}(\zeta)|n\rangle_{a_{1}}|n\rangle_{a_{2}}$, where $S_{12}(\zeta)=\exp \left(\zeta a_{1}^{\dagger} a_{2}^{\dagger}-\zeta^{*} a_{1} a_{2}\right)$ is the two-mode squeezing operator.

If we set $\zeta=|\zeta| e^{i \theta_{\zeta}}$ and introduce the mean photon number per mode $\lambda=\sinh ^{2}|\zeta|$, then $c_{n}(\zeta)=$ $(1+\lambda)^{-1 / 2}\left[\left(1+\lambda^{-1}\right) e^{-i 2 \theta_{\zeta}}\right]^{-n / 2}$. The input modes $b_{1}$ and $b_{2}$ are still excited in two coherent states, so that the four-mode input state is $|\psi\rangle=|\mathrm{TWB}\rangle\rangle_{a_{1}, a_{2}} \otimes|\alpha\rangle_{b_{1}} \otimes|\alpha\rangle_{b_{2}}$. 
If the phase between the TWB and the coherent beams is chosen properly, and for an initial phase shift of the two interferometers close to zero (but not exactly zero), as for example $\phi_{1}=\phi_{2} \sim 0.001$, the use of TWB state leads to the result that the contribution to the uncertainty coming from the photon number noise is $\mathcal{U}_{\mathrm{TWB}}^{(0)} \propto 1 / \lambda$ (when $\mu \gg \lambda \gg 1$ ), representing a great improvement in accuracy of the interferometric scheme.

The question that now arises is how and to which extent our conclusions are affected by an overall non unity transmission-detection efficiency $\eta$ and by consider high order expansion of uncertainty.

The results for this case are summarised in Fig.5b where the ratio of the uncertainties for twin beams with respect to classical inputs are shown as a function of $\eta$. The advantage of the quantum approach is clear, twin beams offering the larger advantage at high $\eta$.

a)

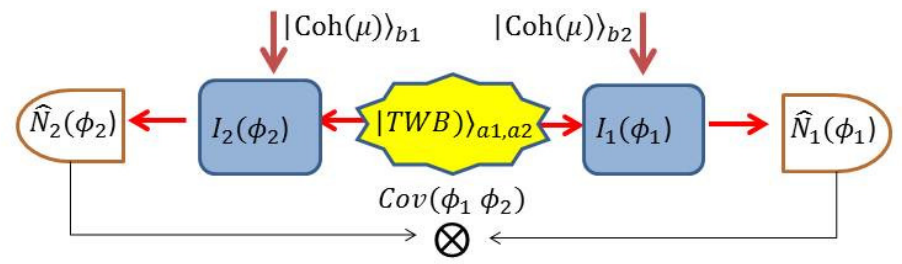

b)

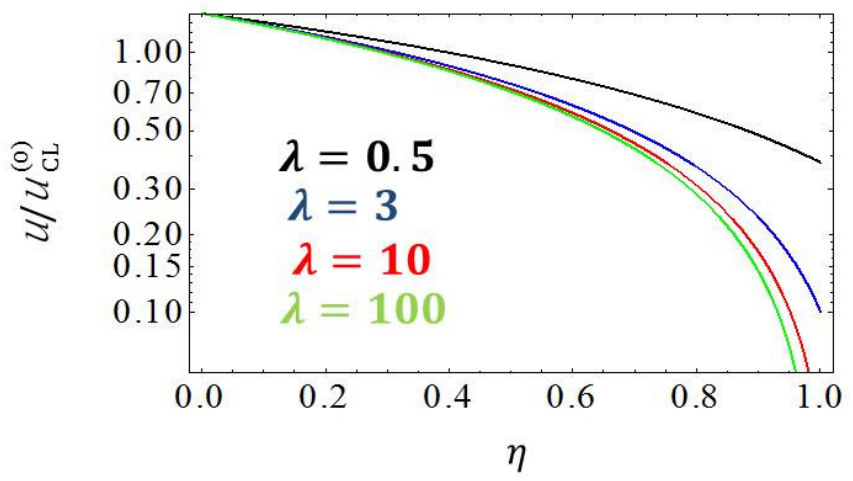

Figure 5. Uncertainty in the determination of the correlation between phase shifts of two interferometers when TWB correlated modes are sent to the unused ports of the two input beam-splitters $\eta$. The Uncertainty is normalized to the value achieved with classical light only. The results are given as a function of the detection efficiency $\eta$, and the different colors represent different intensities of TWB, as described in the legend.

\section{Acknowledgments}

This research was supported by the EU FP7 under grant agreement n. 308803 (BRISQ2), the Compagnia di San Paolo and MIUR (FIRB "LiCHIS" - RBFR10YQ3H, Progetto Premiale "Oltre i limiti classici di misura"); the John Templeton Foundation (the opinions expressed in this publication are those of the authors and do not necessarily reflect the views of the John Templeton Foundation). 


\section{References}

[1] M. Genovese, Research on hidden variable theories: a review of recent progresses, Phys. Rep. 413, 319 (2005)

[2] G. Brida, M. Genovese, and I. Ruo Berchera, Experimental realization of sub-shot-noise quantum imaging, Nature Phot. 4, 227 (2010).

[3] G. Brida, M. Genovese, A. Meda, and I. Ruo Berchera, Experimental quantum imaging exploiting multimode spatial correlation of twin beams, Phys. Rev. A 83, 033811 (2011).

[4] E. Lopaeva, I. Ruo Berchera, I. Degiovanni, S. Olivares, G. Brida, and M. Genovese, Experimental realisation of quantum illumination, Phys. Rev. Lett. 110, 153603 (2013).

[5] E. Lopaeva, I. Ruo Berchera, S. Olivares, G. Brida, I.P. Degiovanni, and M. Genovese, A detailed description of the experimental realization of a quantum illumination protocol, Phys. Scr. T 160, 014026 (2014).

[6] S.-H. Tan, B.I. Erkmen, V. Giovannetti, S. Guha, S. Lloyd, L. Maccone, S. Pirandola, and J.H. Shapiro Quantum illumination with gaussian states, Phys. Rev. Lett. 101, 253601 (2008).

[7] S. Ragy, I. Ruo Berchera, I.P. Degiovanni, S. Olivares, M.G.A. Paris, G. Adesso, and M. Genovese, Quantifying the source of enhancement in experimental continuous variable quantum illumination, J. Opt. Soc. Am. B 31, 2045-2050 (2014).

[8] I. Ruo Berchera, I.P. Degiovanni, S. Olivares, and M. Genovese, Quantum light in coupled interferometers for quantum gravity tests, Phys. Rev. Lett. 110, 213601 (2013).

[9] G. Brida, M. Genovese, A. Meda, I. Ruo Berchera, Experimental quantum imaging exploiting multi-mode spatial correlation of twin beams, Phys. Rev. A 83, 033811 (2011).

[10] G. Brida,M.V. Chekhova, G.A. Fornaro, M. Genovese, L. Lopaeva, and I. Ruo Berchera, Systematic analysis of signal-to-noise ratio in bipartite ghost imaging with classical and quantum light, Phys Rev. A 83, 063807 (2011).

[11] G. Brida, M. Chekhova, M. Genovese, and I. Ruo-Berchera, analysis of the possibility of analog detectors calibration by exploiting stimulated parametric down conversion, Optics Express 16, (2008) 12550; A. Meda, I. Ruo-Berchera, I.P. Degiovanni, G. Brida, M.L. Rastello, and M. Genovese, Absolute calibration of a charge-coupled device camera with twin beams, Appl. Phys. Lett. 105, 101113 (2014).

[12] O. Jedrkiewicz, Y.-K Jiang, E. Brambilla, A. Gatti, M. Bache, L.A. Lugiato, and P. Di Trapani, Detection of Sub-Shot-Noise Spatial Correlation in High-Gain Parametric Down Conversion, Phys. Rev. Lett. 93, 243601 (2004).

[13] J. Peřina, Jr., M. Hamar, V. Michálek, and O. Haderka, Photon-number distributions of twin beams generated in spontaneous parametric down-conversion and measured by an intensified CCD camera Phys. Rev. A 85, 023816 (2012).

[14] M. Bondani, A. Allevi, G. Zambra, M.G.A. Paris, and A. Andreoni, Sub-shot-noise photonnumber correlation in a mesoscopic twin beam of light, Phys. Rev. A 76, 013833 (2007).

[15] T. Iskhakov, M.V. Chekhova, and G. Leuchs, Generation and direct detection of broadband mesoscopic polarization-squeezed vacuum, Phys. Rev. Lett. 102, 183602 (2009).

[16] G. Brida, L. Caspani, A. Gatti, M. Genovese, A. Meda and I. Ruo-Berchera, Measurement of sub-shot-noise spatial correlations without subtraction of background, Phys. Rev. Lett. 102, 213602 (2009).

[17] T.S. Iskhakov, V,C. Usenko, R. Filip, M.V. Chekhova, and G. Leuchs, Low-noise macroscopic twin beams, arXiv: 1408.6407 (2014)

[18] D. Gatto Monticone, K. Katamadze, P. Traina, E. Moreva, J. Forneris, I. Ruo Berchera, P. Olivero, I.P. Degiovanni, G. Brida, and M. Genovese, Beating the Abbe diffraction limit in confocal 
microscopy via nonclassical photon statistics, Phys. Rev. Lett. 113, 143602 (2014).

[19] M.D. Lukin, A.B. Matsko, M. Fleischhauer, and M.O. Scully, Quantum Noise and Correlations in Resonantly Enhanced Wave Mixing Based on Atomic Coherence, Phys. Rev. Lett. 82, 1847 (1999).

[20] C.F. McCormick, A.M. Marino, V. Boyer, and P.D. Lett, Strong low-frequency quantum correlations from a four-wave-mixing amplifier, Phys. Rev. A 78, 043816 (2008).

[21] Q. Glorieux, R. Dubessy, S. Guibal, L. Guidoni, J.-P. Likforman, T. Coudreau, and E. Arimondo, Double- $\lambda$ microscopic model for entangled light generation by four-wave mixing, Phys. Rev. A 82, 033819 (2010)

[22] Q. Glorieux, PhD Thesis, Quantum correlations by four-wave-mixing in atomic vapor. Theory and Experiments, arXiv: 1101.5166 (2011).

[23] R.E. Slusher, L. Hollberg, B. Yurke, J. Mertz and J. Valley, Squeezed states in optical cavities : A spontaneous-emission-noise limit, Phys. Rev. A 31, 3512 (1985).

[24] M. Guo, H. Zhou, D. Wang, J. Gao, J. Zhang, and S. Zhu, Experimental investigation of highfrequency-difference twin beams in hot cesium atoms, Phys. Rev. A 89, 033813 (2014)

[25] S. Zhang, M. Tengner, T. Zhong, F.N.C. Wong, and J.H. Shapiro, Entanglement's benefit survives an entanglement-breaking channel, Phys. Rev. Lett. 111, 010501 (2013); C. Weedbrook, S. Pirandola, J. Thompson, V. Vedral, M. Gu, Discord empowered quantum illumination, arXiv: 1312.3332 (2013).

[26] G. Amelino-Camelia, J. Ellis, N.E. Mavromatos, D.V. Nanopoulos, and S. Sarkar, Tests of quantum gravity from observations of $\gamma$-ray bursts, Nature 393, 763 (1998).

[27] G. Amelino-Camelia, Gravity-wave interferometers as quantum-gravity detectors, Nature 398, 216 (1999).

[28] G. Amelino-Camelia, Astrophysics: Shedding light on the fabric of space-time, Nature 478, 466 (2011).

[29] I. Pikovski, M.R. Vanner, M. Aspelmeyer, M.S. Kim, and Časlav Brukner, Probing Planckscale physics with quantum optics, Nature Phot. 8, 393 (2012); A.Albrecht et al., Tseting quantum gravity by nanodiamond interferometry with nitrogen-vacancy centers, Phys. Rev. A 90, 033834 (2014).

[30] G. Hogan, Interferometers probes of planckian quantum geometry, Phys. Rev. D 85, 064007 (2012).

[31] Fermilab web site www.holometer . fnal .gov (03/23/2012).

[32] J.D. Bekenstein, Is a tabletop search for Planck scale signals feasible?, arXiv:1211.3816 (2012).

[33] P. Aschieri and L. Castellani, Noncommutative gravity solutions, Journ. of Geom. and Phys. 60, 375 (2010).

[34] P. Aschieri and L. Castellani, Noncommutative D=4 gravity coupled to fermions, JHEP 06, 086 (2009).

[35] V. Giovannetti, S. Lloyd, and L. Maccone, Advances in quantum metrology, Nature Phot. 5, 222 (2011).

[36] C.M. Caves, Quantum-mechanical noise in an interferometer, Phys. Rev. D 23, 1693 (1981).

[37] K. McKenzie, D.A. Shaddock, D.E. McClelland, B.C. Buchler, and Ping Koy Lam, Experimental demonstration of a squeezing-enhanced power-recycled michelson interferometer for gravitational wave detection, Phys. Rev. Lett. 88231102 (2002).

[38] The LIGO Scientific Collaboration (R. Schnabel et al.), A gravitational wave observatory operating beyond the quantum shot-noise limit, Nature Phys. 7, 962 (2011). 
[39] The LIGO Scientific Collaboration (L. Barsotti et al.) Enhanced sensitivity of the LIGO gravitational wave detector by using squeezed states of light, Nature Phot. 7, 613 (2013).

[40] S. Steinlechner, J. Bauchrowitz, M. Meinders, H. Müller-Ebhardt, K. Danzmann, and R. Schnabel, Quantum-dense metrology, Nature Phot. 7, 626 (2013). 